Pesq. Vet. Bras. 31(6):465-470, junho 2011

\title{
Intoxicação por Portulaca elatior (Portulacaceae) em caprinos ${ }^{1}$
}

\author{
Glauco J.N. Galiza², Luciano A. Pimentel' ${ }^{2}$, Diego M. Oliveira², Felipe Pierezan ${ }^{3}$, \\ Antônio F.M. Dantas ${ }^{2}$, Rosane M.T. Medeiros ${ }^{2}$ e Franklin Riet-Correa ${ }^{2}$
}

ABSTRACT.- Galiza G.J.N., Pimentel L.A., Oliveira D.M., Pierezan F., Dantas A.F.M., Medeiros R.M.T. \& Riet-Correa F. 2011. [Poisoning by Portulaca elatior (Portulacaceae) in goats.] Intoxicação por Portulaca elatior (Portulacaceae) em caprinos. Pesquisa Veterinária Brasileira 31(6):465-470. Hospital Veterinário, Centro de Saúde e Tecnologia Rural, Universidade Federal de Campina Grande, Campus de Patos, 58700-000 Patos, PB, Brazil. E-mail: franklin.riet@pq.cnpq.br

An outbreak of poisoning by Portulaca elatior was diagnosed in the state of Paraíba, northeastern Brazil, during the rainy season. Between February and April, 16 out of 316 goats were affected and 13 died. Fifteen of the affected animals were from a flock of 16 goats introduced on the farm at the beginning of the rainy season. Clinical signs were intense salivation, reluctance to move, lip tremors, slight uncoordinated gait, diarrhea, bloat, abdominal pain, groans, dehydration and constant bellowing. The course of the disease ranged from 2 to 48 hours; 13 goats died and three recovered. Gross lesions were reddening of the mucosa of rumen and reticulum, hemorrhages in the ruminal serosa, ulcerations of the abomasal mucosa, and ecchymoses in the small intestinal mucosa. Histological examination revealed degeneration and necrosis of epithelial cells with vesicle formation in the fore stomachs associated with infiltration by neutrophils. Suspected to have caused the outbreak, Portulaca elatior was administered experimentally to three animals at doses of 40,20 and 10 grams of the fresh plant per kg bodyweight, respectively. The goats that ingested 20 and $40 \mathrm{~g} / \mathrm{kg}$ died, and the one that ingested $10 \mathrm{~g} / \mathrm{kg}$ showed clinical signs but recovered. These animals showed similar clinical signs and histological lesions as those seen in the goats poisoned spontaneously, demonstrating that the disease was caused by the ingestion of Portulaca elatior. Oxalate concentration were determined in $P$. elatiorand in another plant identified as Blutaparon vermiculare. $P$. elatior contained $6.37 \%$ of total oxalates and $B$. vermiculare $5.29 \%$. B. vermiculare was administered experimentally to one goat at a dose of $40 \mathrm{~g} / \mathrm{kg}$, without causing clinical signs. These results and the absence of oxalate crystals in the digestive system and kidneys of the goats necropsied suggest that poisoning by $P$. elatior is not caused by high oxalate concentration in the plant.

INDEX TERMS: Poisonous plants, Portulaca elatior, Portulacaceae, plant poisoning, goats, digestive system.

RESUMO.- Um surto de intoxicação espontânea por Portulaca elatior foi diagnosticado no município de Aroeiras, Paraíba, na época das chuvas. Entre os meses de fevereiro e abril, em um rebanho de 316 caprinos, 16 adoeceram,

\footnotetext{
${ }^{1}$ Recebido em 16 de dezembro de 2010.

Aceito para publicação em 21 de janeiro de 2011.

2 Centro de Saúde e Tecnologia Rural (CSTR), Universidade Federal de Campina Grande (UFCG), Campus de Patos, 58700-000 Patos, PB, Brasil. *Autor para correspondência: franklin.riet@pq.cnpq.br

${ }^{3}$ Departamento de Patologia, Universidade Federal de Santa Maria, 97105-900 Santa Maria, RS, Brasil.
}

sendo 15 de um lote de 16 caprinos que tinham sido introduzidos recentemente na fazenda e um animal do rebanho já existente na fazenda. Os caprinos apresentaram intensa salivação, relutância em se movimentar, tremores de lábios, marcha incoordenada, diarreia, timpanismo, dor abdominal, gemidos, desidratação e berros constantes. A evolução da enfermidade variou de 2 a 48 horas; 13 caprinos morreram e três se recuperaram. As lesões macroscópicas caracterizavam-se por mucosa ruminal e do retículo avermelhadas, serosa do rúmen hemorrágica, mucosa abomasal avermelhada com áreas ulceradas e mucosa do intesti- 
no delgado com equimoses. No exame histopatológico observou-se degeneração e necrose das células epiteliais dos pré-estômagos com formação de vesículas e infiltrado inflamatório neutrofílico. Suspeita de ter causado o surto, Portulaca elatior foi administrada a três caprinos nas doses de 40, 20 e 10 gramas de planta fresca por kg de peso animal $(\mathrm{g} / \mathrm{kg})$. Esses caprinos apresentaram sinais semeIhantes aos casos espontâneos. Os animais que ingeriram 20 e $40 \mathrm{~g} / \mathrm{kg}$ morreram e o que ingeriu $10 \mathrm{~g} / \mathrm{kg}$ se recuperou. Microscopicamente foram observadas lesões do sistema digestivo semelhantes às dos casos espontâneos. A reprodução experimental da intoxicação, com sinais clínicos e lesões histológicas semelhantes às dos casos espontâneos, comprova que a doença foi causada por Portulaca elatior. $P$. elatior apresentou níveis de oxalatos de $6,37 \%$ e outra planta, identificada como Blutaparon vermiculare e que ocorria em grande quantidade nas pastagens, apresentou níveis de oxalatos de $5,29 \%$. B. vermiculare foi administrada experimentalmente a um caprino na dose de $40 \mathrm{~g} / \mathrm{kg}$, sem que o animal apresentasse sinais clínicos. Esse resultado e a ausência de cristais de oxalatos no sistema digestivo e rins dos caprinos necropsiados, sugerem que a intoxicação por $P$. elatior não é causada pela alta concentração de oxalatos na planta.

TERMOS DE INDEXAÇÃO: Plantas tóxicas, Portulaca elatior, Portulacaceae, intoxicação por planta, caprinos, sistema digestivo.

\section{INTRODUÇÃO}

O gênero Portulaca apresenta cerca de 115 espécies no mundo (Eggli \& Ford-Werntz 2002) e no Brasil foram relatadas 13 espécies (Coelho \& Giulietti 2010). Diversas espécies desse gênero são descritas no Nordeste brasileiro, sendo que Portulaca elatior é encontrada nos municípios de Juazeiro e Castro Alves, Estado da Bahia, e no município de Petrolândia, Estado do Pernambuco. Portulaca oleracea é encontrada na microrregião do Seridó Oriental e Ocidental do Rio Grande do Norte. Essas espécies têm sido relatadas pelos produtores rurais, como causa de manifestações clínicas de perturbações gastrintestinais em bovinos dessas regiões (Carvalho \& Marchini 1999, Durval et al. 2006, Albuquerque et al. 2007, Silva et al. 2009, Coelho et al. 2010). Portulaca spp. e $P$. oleracea contêm altas concentrações de oxalatos solúveis (Radostits et al. 2007).

Na Região Nordeste são conhecidas atualmente seis espécies de plantas tóxicas que afetam o sistema digestivo dos ruminantes: Enterolobium contortisiliquum (Tokarnia et al. 1999, Benício et al. 2007) Stryphnodendron coriaceum (Tokarnia et al. 1991) e Luetzelburgia auriculata que causam alterações digestivas com lesões degenerativas dos pré-estômagos e diarreia ou fezes amolecidas (Cavalcanti 1995, Mello et al. 2010); Arrabidaea corallina que cursa com diarreia (Pessoa et al. 2010); Plumbago scandens causa degeneração e necrose da mucosa dos pré-estômagos com pigmentação da urina e mucosa do trato digestivo (Tokarnia \& Döbereiner 1975, 1976, Medeiros et al. 2001); Centratherum brachylepis que causa degeneração e necrose dos pré-estômagos com edema de face e garganta (Medeiros et al. 2009) e Dieffenbachia spp. que cursam com edema de língua e face (Dantas et al. 2007).

O objetivo deste trabalho é relatar um surto de intoxicação espontânea por Portulaca elatior em caprinos, abordando seus aspectos epidemiológicos, clínicos e patológicos, além da reprodução experimental da intoxicação.

\section{MATERIAL E MÉTODOS}

Quatro caprinos intoxicados espontaneamente, três mortos e um doente, foram encaminhados para o Hospital Veterinário (HV) da Universidade Federal de Campina Grande, Campus de Patos, Paraíba. Do caprino doente foi realizado exame clínico e amostras de sangue e urina foram coletadas para realização de hemograma, urinálise e determinação das atividades séricas de aspartato-amino transferase (AST) e gamaglutamil transferase (GGT).

Dados epidemiológicos e clínicos foram obtidos em visitas à propriedade onde ocorreram os casos de intoxicação. Após observação da área onde os animais eram criados, verificouse que das plantas encontradas na propriedade, uma delas ainda não conhecida como tóxica, tinha sido ingerida pelos caprinos. Amostras dessa planta e de outra planta presente em grande quantidade, que por ser uma Amaranthaceae era suspeita de conter oxalatos em alta quantidade, foram coletadas e enviadas ao professor Odací F. de Oliveira, Universidade Federal Rural do Semi-Árido (UFERSA), para identificação botânica, e para a professora Valíria D. Cerqueira, Universidade Federal do Pará, para determinação dos níveis de oxalatos totais de acordo com a técnica utilizada por Ribeiro et al. (2006).

Para a reprodução experimental da intoxicação, foi coletada a planta suspeita de causar a intoxicação, posteriormente identificada como Portulaca elatior Mart. ex Rohrb (fam. Portulacaceae) (Fig.1), e administrada a três caprinos da raça Moxotó, machos com idade de 6 a 18 meses, pesando de 8,1-14,8 kg. Os animais foram alojados em baias individuais e pesados após jejum de 12 horas, identificados e vermifugados. A planta coletada na propriedade onde estavam ocorrendo as intoxicações foi conservada refrigerada, sendo posteriormente administrada manualmente por via oral, em doses únicas de 10, 20 e 40g/kg. Os movimentos ruminais e as frequências cardíacas e respiratórias foram aferidos antes e ao final da administração e diariamente pela manhã, tarde e noite após a ingestão da planta. No dia seguinte, após a administração da planta, os animais foram alimentados com feno de capim Tifton (Cynodon dactylon) à vontade e concentrado, constituído por ração comercial, farelo de trigo e farelo de milho, em quantidade equivalente a $1 \%$ do peso do animal.

Quatro caprinos afetados espontaneamente e dois caprinos intoxicados experimentalmente foram necropsiados. Fragmentos de órgãos das cavidades torácica e abdominal e do sistema nervoso central foram coletados, fixados em formol tamponado a 10\%, incluídos em parafina, cortados a 4-6 micras e corados pela hematoxilina e eosina $(\mathrm{HE})$, para exame histopatológico.

Outra planta, identificada como Blutaparon vermiculare (L.) Mears (Amaranthaceae), encontrada em maior quantidade que $P$. elatior e que era consumida normalmente pelos animais, foi coletada e administrada na dose de $40 \mathrm{~g} / \mathrm{kg}$ de peso animal a um caprino jovem. 

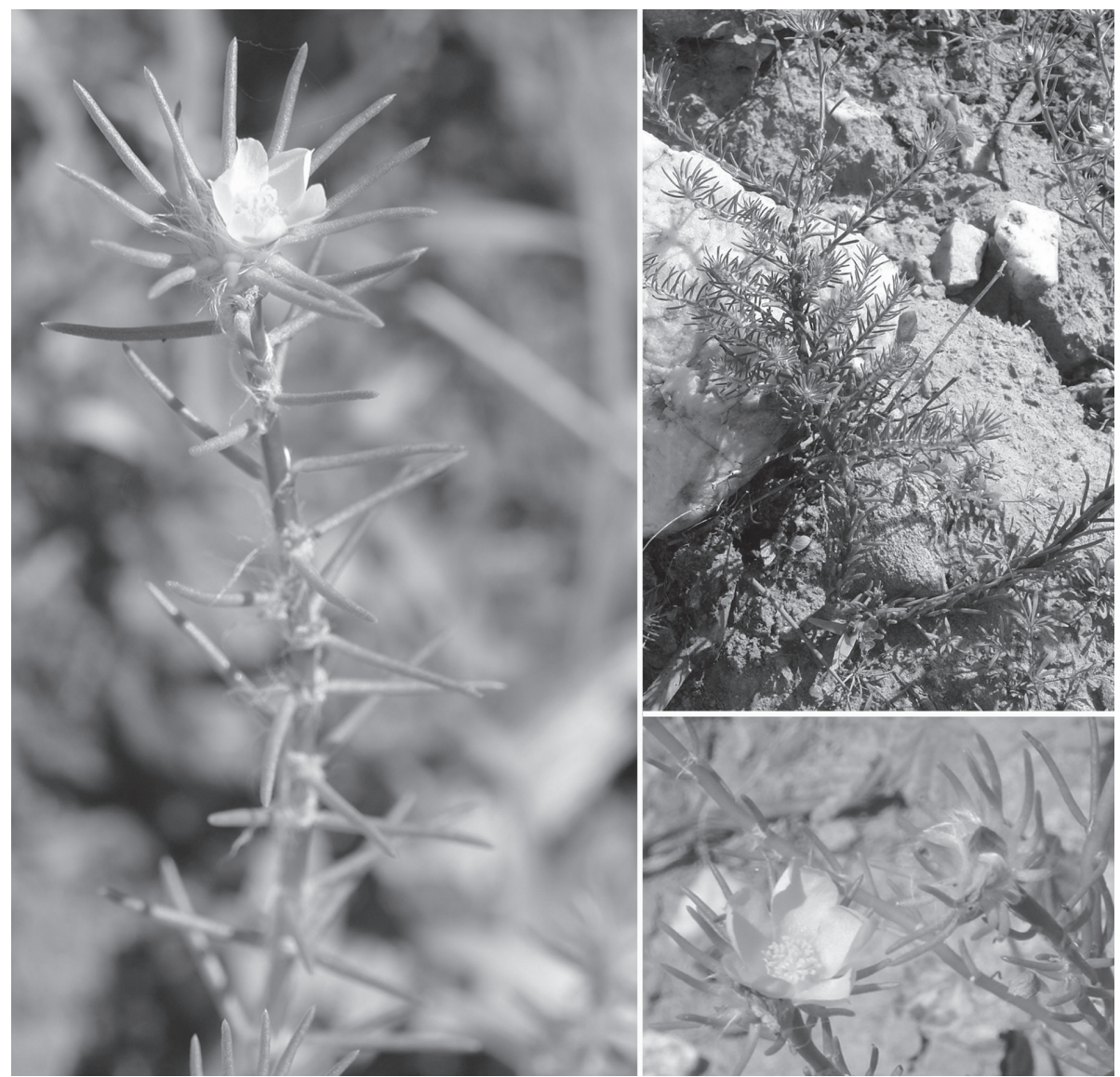

Fig.1. Portulaca elatior no município de Aroeiras, Paraíba.

\section{Intoxicação espontânea}

\section{RESULTADOS}

O surto ocorreu em uma propriedade no município de Aroeiras $\left(07^{\circ} 322433 S\right.$ e $\left.35^{\circ} 422273 \mathrm{~W}\right)$, no Agreste da Paraíba. O rebanho era composto por 316 caprinos de diversas idades, sendo que 16 fêmeas, jovens e adultas, tinham sido adquiridas recentemente e introduzidas na propriedade após o início do período chuvoso, em fevereiro. Durante os meses de fevereiro a abril de 2008, dos 16 caprinos recém introduzidos, adoeceram 15 (93,7\%), 12 (80\%) morreram e três se recuperam. Dos 300 caprinos existentes previamente na propriedade, apenas um caprino foi afetado e morreu. Todos os animais eram vermifugados e vacinados contra raiva e clostridioses. Os caprinos eram mantidos nas pastagens durante o dia e encerrados em currais à noite.

Os sinais clínicos observados nos caprinos foram intensa salivação, relutância em se movimentar, tremores de lábios, marcha levemente incoordenada arrastando as pinças dos membros posteriores, diarreia, timpanismo, dor abdominal, gemidos, desidratação e berros constantes. A morte ocorria 2 a 48 horas após o início dos sinais clínicos. Em um animal intoxicado não foram observadas alterações no hemograma, urinálise e atividades séricas de AST e GGT.

As alterações macroscópicas eram restritas ao trato digestivo, principalmente dos pré-estômagos. As papilas do rúmen estavam avermelhadas (Fig.2A). Em um dos caprinos observaram-se hemorragias na serosa dos sacos cego caudo-dorsal e cego caudo-ventral do rúmen (Fig.2B). Observou-se também avermelhamento discreto da mucosa do retículo e do abomaso, além de múltiplas ulcerações irregulares na mucosa do abomaso. No intestino delgado havia avermelhamento da mucosa e equimoses. Dos outros dois animais, apenas um apresentava alterações intestinais semelhantes ao caso anterior e no outro caprino não foram observadas alterações macroscópicas significativas.

Microscopicamente observaram-se, nos pré-estômagos, áreas multifocais de vacuolização das células epiteliais da mucosa com formação de vesículas, algumas contendo raros neutrófilos no seu interior (Fig.3A). No rúmen de um dos animais havia vacuolização difusa moderada do epitélio e hiperemia na submucosa (Fig.3B). Ocasionalmente, principalmente nas áreas próximas às vesículas, havia células picnóticas e eosinofílicas, características de necrose individual. Na submucosa observaram-se também áreas multifocais com moderado infiltrado inflamatório predominantemente constituído por neutrófilos, associado à hiperemia e discreta hemorragia. Essas alterações eram moderadas no omaso e discretas no rúmen e no retículo. No intestino delgado observou-se moderado infiltrado inflamatório mononuclear causando discreta distensão da 


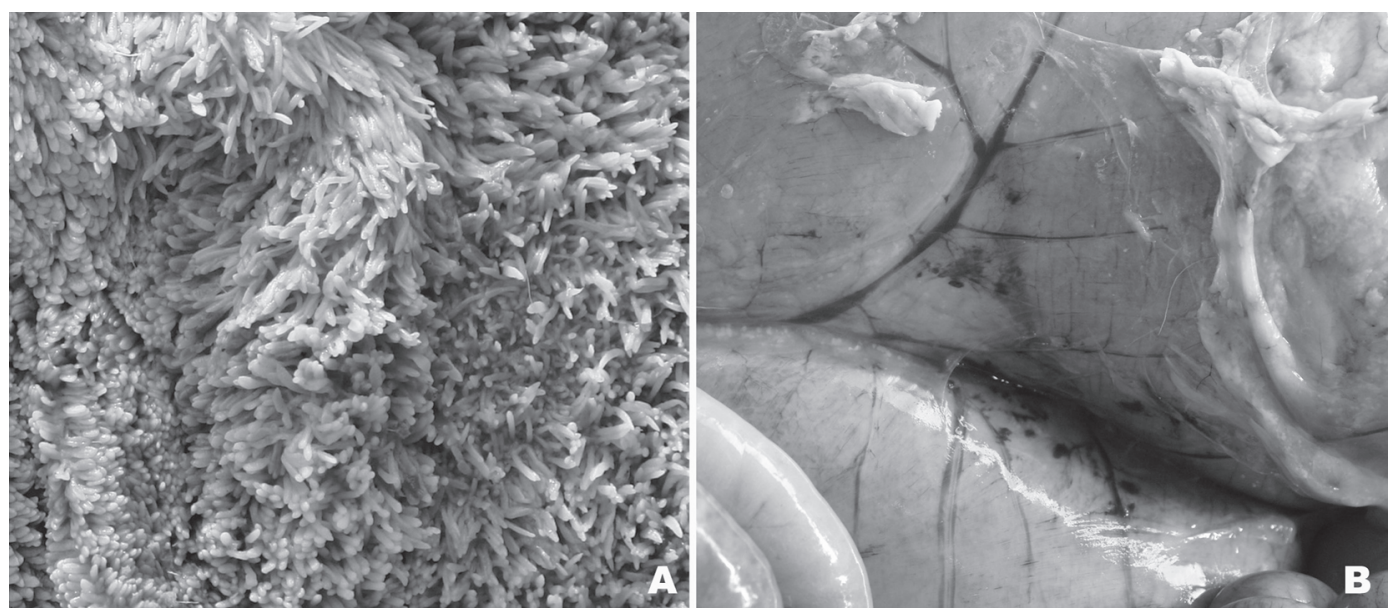

Fig.2. (A) Rúmen de caprino intoxicado espontaneamente por Portulaca elatior com avermelhamento da mucosa ruminal e (B) áreas de hemorragia na serosa dos sacos cegos caudo-dorsal e caudo-ventral.

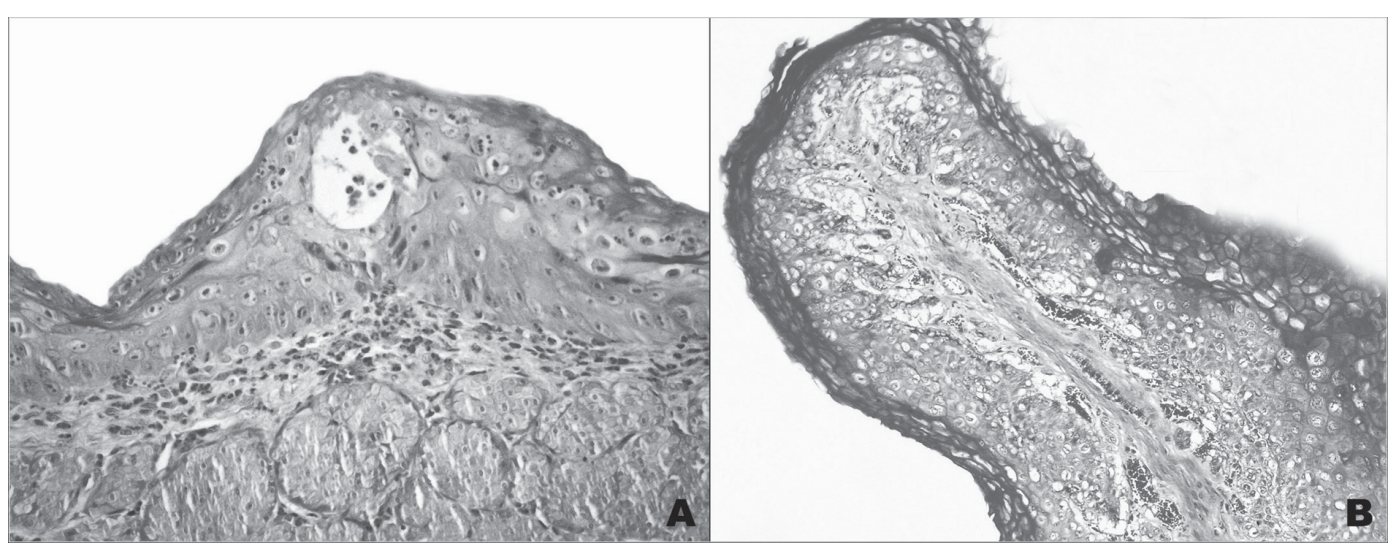

Fig.3. (A) Omaso de caprino intoxicado espontaneamente por Portulaca elatior apresentando vacuolização das células epiteliais da mucosa e formação de vesícula com alguns neutrófilos. $\mathrm{Na}$ mucosa observa-se moderado infiltrado inflamatório de neutrófilos. HE, obj.40x. (B) Rúmen de caprino intoxicado espontaneamente por Portulaca elatior apresentando vacuolização difusa moderada da mucosa e hiperemia na submucosa. HE, obj.20x.

lâmina própria, associado à discreta hiperemia. Não foram observadas alterações microscópicas nos outros órgãos.

As concentrações de oxalatos totais foram de $6,37 \%$ nas amostras de Portulaca elatior e 5,29\% nas de Blutaparon vermiculare.

\section{Intoxicação experimental}

O Caprino 1, intoxicado experimentalmente com $40 \mathrm{~g} /$ $\mathrm{kg}$ de peso animal de Portulaca elatior, apresentou, 63 horas após a administração da planta, balançar excessivo de cauda e relutância para se movimentar permanecendo em estação com membros abertos. Quando agitado apresentava tremores generalizados evoluindo para decúbito esternal e lateral. Outros sinais incluíam tremores de intenção, taquicardia, micção constante, timpanismo, dor abdominal, berros constantes, ausência de reflexo pupilar, movimentos de pedalagem, trismo mandibular, ptose labial, nistagmo e convulsões de aproximadamente 30 segundos de duração com intervalos de cerca de um minuto.
Durante as convulsões apresentava espasticidade, rotação ocular, opistótono e salivação espumosa. O animal morreu após 9 horas do início dos sinais clínicos.

O Caprino 2, intoxicado com $20 \mathrm{~g} / \mathrm{kg}$ de $P$. elatior, apresentou, 37 horas após a administração, apatia, leves tremores de orelha e cabeça, relutância em se movimentar e retração do lábio inferior. Quando forçado a caminhar apresentava decúbito esternal estendendo a cabeça e pescoço, salivação espumosa discreta, bruxismo e diarreia. Quando agitado os tremores de orelha e cabeça se intensificavam. Os sinais evoluíram para decúbito lateral e quando colocado em estação caminhava pouco e caia e não conseguia se levantar, apresentando berros constantes, contrações musculares seguidas de espasticidade moderada, movimentos de pedalagem, nistagmo, estrabismo medial, torcicolo e, finalmente, estado comatoso com dilatação das pupilas. Após uma evolução clínica de 78 horas o animal foi eutanasiado in extremis.

O Caprino 3 , que recebeu $10 \mathrm{~g} / \mathrm{kg}$ de $P$. elatior, apre- 
sentou, 44 horas após o fim da administração, apatia, relutância em se movimentar, discreta salivação espumosa, bruxismo, diarreia e decúbito esternal. Quando estimulado levantava-se e permanecia em estação. Os sinais clínicos regrediram e, 48 horas após o início desses sinais, o animal recuperou-se.

O Caprino 4 , que recebeu $40 \mathrm{~g} / \mathrm{kg}$ de Blutaparon vermiculare, não apresentou nenhuma alteração clínica.

Nos caprinos intoxicados experimentalmente não foram observadas alterações macroscópicas significativas. Microscopicamente evidenciou-se, nos pré-estômagos, discreta vacuolização das células epiteliais da mucosa e, em algumas áreas, essa vacuolização ocorria também nas células epiteliais do estrato basal. Ainda havia discreto edema intercelular (espongiose) e necrose individual das células epiteliais do retículo. Na submucosa havia áreas multifocais de discreto infiltrado inflamatório mononuclear com raros neutrófilos associado à hiperemia. Essas alterações eram observadas principalmente no omaso, além de rúmen e retículo. Não foram observadas lesões microscópicas significantes nos demais órgãos.

\section{DISCUSSÃO}

O diagnóstico de intoxicação por Portulaca elatior foi baseado nos achados epidemiológicos, clínicos e patológicos, associados à reprodução experimental da doença.

Apesar do elevado nível de oxalatos totais presentes em $P$. elatior $(6,37 \%)$, sugere-se que o oxalato não esteja envolvido na patogênese da intoxicação, visto que $B$. vermiculare também apresenta níveis elevados de oxalatos $(5,29 \%)$ e não causou quadro clínico quando administrada a $40 \mathrm{~g} / \mathrm{kg}$, enquanto que $P$. elatior causou sinais clínicos na dose de $10 \mathrm{~g} / \mathrm{kg}$ e morte dos animais nas doses de $20 \mathrm{e}$ $40 \mathrm{~g} / \mathrm{kg}$. Além disso, não foram encontrados cristais de oxalatos nos rins nem no sistema digestivo.

No presente surto de intoxicação por $P$. elatior evidenciou-se uma alta morbidade $(93,7 \%)$ e mortalidade $(80 \%)$ nos caprinos introduzidos recentemente na propriedade, enquanto que dos 300 caprinos que estavam na fazenda anteriormente somente um foi afetado. Isso sugere que a intoxicação ocorre, preferencialmente, em animais transportados com desconhecimento prévio da planta. Para o controle dessa intoxicação é necessário evitar que animais que não conheçam a planta, sejam colocados em áreas onde exista grande disponibilidade de $P$. elatior, principalmente durante o período chuvoso.

O quadro clínico agudo e a epidemiologia dessa intoxicação devem ser diferenciados das plantas que causam desordens digestivas no semiárido citadas na introdução. Os sinais clínicos e lesões observadas comprovam que é uma planta que afeta primariamente o sistema digestivo. A observação de sinais nervosos sugere que os mesmos podem estar associados às alterações agudas no trato digestivo, como observado nos casos de intoxicação por Baccharis spp. (Tokarnia \& Döbereiner 1975, 1976, Armién et al. 1993, Barbosa et al. 1994, Rissi et al. 2005, Alda et al. 2009) e Phytolacca decandra (Peixoto et al. 1997, Ecco et al. 2001).
Novos estudos são necessários para determinar o princípio ativo da planta e a patogenia da intoxicação.

Conclui-se que Portulaca elatior causa intoxicação em caprinos no semiárido afetando principalmente caprinos introduzidos recentemente em áreas invadidas pela planta. Os produtores de caprinos da região devem ser orientados a evitar o acesso de animais recém adquiridos em áreas com grande quantidade de $P$. elatior.

Agradecimentos.- Ao professor Odací F. de Oliveira, Universidade Federal Rural Semiárido, pela identificação botânica das plantas e à professora Valíria D. Cerqueira, Universidade Federal do Pará, pela determinação de oxalatos. Este trabalho foi financiado pelo Instituto Nacional de Ciência e Tecnologia para o Controle das Intoxicações por Plantas (CNPq, Proc.573534/2008-0). O primeiro autor agradece ao $\mathrm{CNPq}$ e à CAPES pela concessão de bolsas de iniciação científica e de mestrado respectivamente.

\section{REFERÊNCIAS}

Albuquerque U.P., Medeiros P.M., Almeida A.L.S., Monteiro J.M., Neto E.M.F.L., Melo J.G. \& Santos J.P. 2007. Medicinal plants of the caatinga (semi-arid) vegetation of NE Brazil: A quantitative approach. J. Ethnopharmacol. 114:325-354.

Alda J.L., Sallis E.S.V., Nogueira C.E.W., Soares M.P., Amaral L., Marcolongo-Pereira C., Xavier F., Frey Júnior F. \& Schild A.L. 2009. Intoxicação espontânea por Baccharis coridifolia (Compositae) em equinos no Rio Grande do Sul. Pesq. Vet. Bras. 29:409-414.

Armién A.G., Peixoto P.V. \& Tokarnia C.H. 1993. Intoxicação experimental por Baccharis megapotamica var. megapotamica e var. weirii (Compositae) em ovinos. Pesq. Vet. Bras. 13:5-20.

Barbosa J.D., Armién A.G. \& Tokarnia C.H. 1994. Intoxicação experimental por Baccharis megapotamica var. weirii (Compositae) em caprinos. Pesq. Vet. Bras. 14:5-13.

Benício T.M.A., Nardelli M.J., Nogueira F.R.B., Araújo J.A.S. \& RietCorrea. 2007. Intoxication by the pods of Enterolobium contortisiliquum in goats, p.514-519. In: Panter K.E., Wirenga T.L. \& Pfister J.A. (Eds), Poisonous Plants: Global research and solutions. CABI Publishing, Wallingford, Oxon, UK.

Carvalho C.A.L \& Marchini L.C. 1999. Plantas visitadas por Apis mellifera L. no vale do rio Paraguaçu, Município de Castro Alves, Bahia. Revta Bras. Bot. 22:333-338.

Cavalcanti M.V.L. 1995. Intoxicação experimental em caprinos pela fava do pau mocó (Luetzelburgia sp). Monografia, Centro de Ciências Agrárias, Universidade Federal do Piauí, Teresina. 21p.

Coelho A.A.O.P. \& Giulietti A.M. 2010. O gênero Portulaca L. (Portulacaceae) no Brasil. Act. Bot. Bras. 24:655-670.

Coelho A.A.O.P., Giulietti A.M., Harley R.M. \& Yesilyurt J.C. 2010. Synonymies and typifications in Portulaca (Portulacaceae) of Brazil. Kew Bulletin 65:37-43.

Dantas A.C., Guimarães J.A., Câmara A.C.L., Afonso J.A.B., Mendonça C.L., Costa N.A. \& Souza M.I. 2007. Intoxicação natural por comigo-ninguém-pode (Dieffenbachia sp.) em caprino. Ciênc. Vet. Trop. 10:119-123.

Durval M.S., Riet-Correa F., Medeiros R.M.T. \& Oliveira O.F. 2006. Plantas tóxicas para ruminantes e eqüídeos no Seridó Oriental e Ocidental do Rio Grande do Norte. Pesq. Vet. Bras. 26:223-236.

Ecco R., Barros C.S.L. \& Irigoyen L.F. 2001. Intoxicação experimental por Phytolacca decandra em ovinos. Ciência Rural 31:319-322.

Eggli U. \& Ford-Werntz D. 2002. Illustrated Handbook of Succulent Plants: Dicotyledons, Portulacaceae. Springer, New York, p.370432.

Medeiros R.M.T., Barbosa R.C., Lima E.F., Simões S.V.S. \& Riet- 
Correa F. 2001. Intoxication by Plumbago scandens in goats in $\mathrm{Pa}-$ raíba, northeastern Brazil. Vet. Hum. Toxicol. 43(3):167-169.

Medeiros R.M.T., Yassaki J.K.M., Araújo J.A., Dantas A.F.M. \& RietCorrea F. 2009. Poisoning by Centraterum brachylepis in ruminants. Toxicon 54(1):77-79.

Mello G.W.S., Oliveira D.M., Carvalho C.J.S., Cavalcante M.V.F.L., Costa F.A.L., Riet-Correa F. \& Silva S.M.M.S. 2010. Poisoning of goats by the pods of Luetzelburgia auriculata. Toxicon 55:11151118.

Peixoto P.V., Wouters F., Lemos R.A. \& Loretti A.P. 1997. Phytolacca decandra poisoning in sheep in southern Brazil. Vet. Hum. Toxicol. 39(5):302-303..

Pessoa C.R.M., Medeiros R.M.T., Pessoa A.F.A., Araújo J.A., Dantas A.F.M., Silva-Castro M.M. \& Riet-Correa F. 2010. Diarréia em caprinos associada ao consumo de Arrabidaea corallina (Bignoniaceae). Pesq. Vet. Bras. 30:547-550.

Radostits O.M., Clay C.C., Hinchcliff K.W. \& Constable P.D. 2007. Veterinary Medicine. Saunders Elsevier, Edinburgh, p.1858-1860.

Ribeiro C.B., Medeiros S.R., Macedo M.C.M. \& Morais M.G. 2006. Teores de oxalato nas principais forrageiras utilizadas no Brasil e sua implicação na toxidez em pastejo. $2^{\mathrm{a}}$ Jornada Científica da Embrapa Gado de Corte, 18-20 out., Campo Grande, Mato Grosso do Sul, Brasil. Disponível em <http://www.cnpgc.embrapa.br/eventos/
2006/jornada2/TrabalhosProntos_Final_PDF/MS/046ribeirocb.pdf> Acessado em 11 set. 2010.

Rissi D.R., Rech R.R., Fighera R.A., Cagnini D.Q., Kommers G.D. \& Barros C.S.L. 2005. Intoxicação espontânea por Baccharis coridifolia em bovinos. Pesq. Vet. Bras. 25:111-114.

Silva K.A., Araújo E.L. \& Ferraz E.M.N. 2009. Estudo florístico do componente herbáceo e relação com solos em áreas de caatinga do embasamento cristalino e bacia sedimentar, Petrolândia, PE, Brasil. Acta Bot. Bras. 23:100-110.

Tokarnia C.H. \& Döbereiner 1975. Intoxicação experimental em bovinos por "mio-mio", Baccharis coridifolia. Pesq. Agropec. Bras., Sér. Vet. 10:79-97.

Tokarnia C.H. \& Döbereiner J. 1976. Intoxicação experimental em ovinos por "mio-mio", Baccharis cordifolia. Pesq. Agropec. Bras., Sér. Vet. 11:19-26.

Tokarnia C.H., Peixoto P.V., Gava A. \& Döbereiner J. 1991. Intoxicação experimental por Stryphnodendron coriaceum (Leg. Mimosoideae) em bovinos. Pesq. Vet. Bras. 11:25-29

Tokarnia C.H., Döbereiner J., Dutra I.S., Brito I.S., Chagas B.R., França T.N. \& Brust L.A.G. 1999. Experimentos em bovinos com favas de Enterolobium contortisiliquum e E. timbouva para verificar propriedades fotossensibilizante e/ou abortivas. Pesq. Vet. Bras. 19:39-45 